\title{
Agentivité et citoyenneté linguistique de la francophonie en Ontario
}

\author{
Fanny Macé \\ University of Calgary \\ Canada
}

\section{Mise en contexte, définitions et cadre théorique}

Le Canada, pays officiellement bilingue, compte au premier trimestre 2020 environ 37,9 millions d'habitants (Statistique Canada, 2020). Au sein de cette population, moins d'un tiers - soit à peu près 10 millions - est identifié comme francophones ${ }^{1}$. Au Canada, les francophones vivant en situation minoritaire (hors Québec) sont répartis inégalement à travers les dix provinces et les trois territoires, représentant 2,7 millions de locuteurs. Dans ce contexte, la province de l'Ontario, située entre le Québec à l'Est et le Manitoba à l'Ouest, compte le plus d'habitants au Canada, avec à elle seule plus du tiers de la population canadienne totale, soit un peu plus de 14,7 millions d'habitants. De même, l'Ontario compte la plus importante communauté francophone en situation minoritaire au pays, soit 622415 personnes ou encore $4,7 \%$ de la population ontarienne (Gouvernement de l'Ontario, 2019). Bien que le nombre de francophones en Ontario ait sensiblement augmenté depuis le recensement de 2011, passant de 611500 (recensement de 2011) à 622 415 (recensement de 2016), la proportion de francophones par rapport à la population ontarienne totale continue de diminuer depuis 1986. De plus, les chiffres de l'Ontario révèlent un pourcentage de sa population francophone inférieur à $5 \%$, ce qui dépeint une précarité linguistique et sociale exacerbée par une dispersion inouïe sur un territoire géographique immense; la situation linguistique est en effet très disparate puisque l'on relève que $22,6 \%$ de la population francophone habite dans le Nord-est de la province, soit presque une personne sur quatre, que $15,4 \%$ de cette population se répartit dans l'Est, soit environ une personne sur six et que dans le reste de l'Ontario, la population francophone représente moins de $5 \%$ de la population totale. Comme cela s'avère souvent être le cas dans d'autres contextes francophones minoritaires canadiens, des poches francophones subsistent ainsi en Ontario, en particulier à Penetanguishene, à Welland, dans l'Outaouais, ainsi qu'à Sudbury et Hearst. Cependant, si on y inclut les contextes urbains, à l'échelle provinciale, la communauté francophone ontarienne se distingue principalement par son éparpillement et son manque de visibilité en particulier dans la sphère publique. À ce propos, James Scott (2009) nous rappelle en effet que les occasions d'entendre publiquement la voix des minorités s'avèrent relativement peu nombreuses et que ces moments ont principalement lieu dans des espaces peu accessibles aux chercheur.e.s. Ainsi, ces voix inaudibles parce que distantes ou peu à l'unisson peuvent-elles rejaillir sous des formes inattendues. Dans cette perspective, la situation sociodémographique des francophones minoritaires nous amène à nous questionner sur la notion de droit linguistique. S'agit-il d'un paradigme toujours approprié et équitable lorsqu'il s'agit de faire entendre les voix des minorités linguistiques? Dans ce contexte, chercher une forme de légitimité par la seule application de droits linguistiques permet-il de répondre de manière optimale aux attentes des communautés canadiennes de langues officielles minorisées? Y-aurait-il une solution alternative qui permettrait à la fois d'embrasser les revendications des minorisé.e.s tout en répondant à la problématique de leur manque de visibilité?

Dans le cadre de ce questionnement et afin d'illustrer notre propos, nous avons choisi de revisiter un courtmétrage produit en 1987 par l'Office National du Film du Canada (ONF), « Deux voix, comme en écho » de Claudette Jaïko et de soumettre cet exemple du cinéma identitaire franco-ontarien à l'analyse sociolinguistique en utilisant une grille théorique basée sur les notions de citoyenneté linguistique (Lim et al., 2018; Stroud, 2001), de voix - au sens de donner une voix - (Bell, 2017) et d'agentivité (Emirbayer et Mische, 1998). Il convient de signaler que Serge Miville, dans un article de 2013, a proposé une analyse de ce film identitaire - ainsi que trois autres - selon un cadre théorique reprenant le concept de " fatigue culturelle » développé par Hubert Aquin dans son essai de 1962 et dans lequel il définissait cette dernière comme un phénomène d'attirance-répulsion entre la société dominante (culture anglophone canadienne) et la société dominée (minorité canadienne-française). La lecture de cet article de Miville (2013) et l'analyse qui en découle nous a incités à reconsidérer la problématique identitaire franco-ontarienne sous un jour nouveau. Le choix s'est donc naturellement porté sur ce documentaire : d'abord, parce qu'il est révélateur des tensions relatives aux processus d'identification et d'auto-catégorisation des Franco-ontariens et ensuite parce qu'il permet une certaine conscientisation de l'idéologie monolingue.

Dans ce contexte, le concept de « citoyenneté linguistique » (linguistic citizenship ou LC) tel que défini par Christopher Stroud (2001) et développé par Lim et al. (2018) nous parait approprié dans la mesure où nous l'envisageons comme « un modèle conceptuel dans lequel penser différemment (...) à propos de la langue et de nous-mêmes (p. 3), (...) qui pourrait comprendre une politique linguistique propice à l'action et au changement » (p. 18) (Lim et al., 2018, p. 3 et p. 18, je traduis ${ }^{2}$ ). Ainsi, Stroud (2001) nous invite à 
" impenser » (Sing et Dansereau, 2012) nos définitions préconçues et stéréotypées de langue et de citoyenneté en déconstruisant les voix dominantes notamment « à travers le prisme de la citoyenneté et de la démocratie participative (...)» (Stroud, 2001, p. 18, je traduis ${ }^{3}$ ). Ce faisant, Stroud (2001) plaide pour « des formes alternatives d'être ensemble dans la différence (...) [en] reconstruisant et [en favorisant] les rencontres multilingues comme des moments de célébration de la différence plutôt que de suppression de la voix » (p. 37, je traduis ${ }^{4}$ ). Si nous nous tournons à présent vers le concept de "voix », force est de constater que nous pouvons l'envisager sous divers angles : comme la caractéristique de la personne qui parle - devenant par cette action même agente de la parole, du langage - ou encore sous forme de collectif - il s'agit dans ce cas de la pensée d'une collectivité revendiquée. Par contraste, on utilise aussi le collectif « sans voix » afin de définir un peuple, une collectivité, une minorité qui n'est pas/plus en mesure de se faire entendre, de s'exprimer, qui a été d'une manière ou d'une autre réduit.e au silence. Enfin, selon Bell (2017, p. 588), « donner une voix à quelqu'un » (giving voice) peut ainsi s'appliquer à deux contextes différents : d'une part, il peut s'agir de s'octroyer sa propre voix en exprimant ce que l'on a à dire ou, d'autre part, de permettre à l'Autre de faire entendre son point de vue et d'ainsi obtenir une voix qui lui est propre. C'est cette dernière définition de Bell (2017) - « donner une voix à quelqu'un » (giving voice) - sur laquelle s'appuiera l'analyse de "Deux voix, comme en écho ». Ce faisant, nous tâcherons de mettre l'accent sur ce qu'un.e acteur.trice social.e a à dire ainsi que sur la question du degré d'agentivité nécessaire à la performance de l'acte de citoyenneté linguistique (Stroud, 2001; Lim et al., 2018). Mais qu'entend-on au juste par agentivité? Selon, Mustafa Emirbayer et Ann Mische (1998, p. 962), l'agentivité humaine se définit

comme un processus d'engagement social ancré dans le temps, informé par le passé (dans son aspect « itérationnel » ou habituel) mais aussi orienté vers l'avenir (comme capacité " projective » d'imaginer des possibilités alternatives) et vers le présent (comme "capacité évaluative-pratique » à conceptualiser les habitudes passées et les projets futurs dans les contingences du moment). ${ }^{5}$

Ces deux derniers auteurs proposent donc de concevoir l'agentivité sociale comme située temporellement. Ainsi, les acteurs.trices sociaux.les peuvent adopter diverses orientations agentiques simultanées leur permettant d'évoluer à travers différents contextes temporels. Ils sont ainsi en mesure de s'orienter à la fois vers le passé, le futur et le présent mais peuvent aussi se diriger principalement vers l'un ou l'autre de ces éléments lors d'une situation spécifique (Emirbayer et Mische, 1998, p. 964). Comme cela est clairement exprimé par les auteurs, le fait de changer de perspective en ce qui a trait à l'agentivité permet d'« obtenir un levier analytique crucial pour cartographier les divers degrés de maniabilité, d'inventivité et de choix réflexif des acteurs sociaux en relation avec les contextes d'action contraignants et favorables » (Emirbayer et Mische, 1998, p. 964; je traduis $\left.{ }^{6}\right)$. En bonne héritière des Lumières et des courants utilitaristes ${ }^{7}$, la notion d'agentivité sociale est intimement liée à l'idée d'individualisme et de libre-arbitre : "la capacité qu'ont les êtres humains à façonner les circonstances dans lesquelles ils vivent » (Emirbayer et Mische, 1998, p. 965; je traduis ${ }^{8}$ ). En ce sens, cette définition particulière de l'agentivité nous permettra de travailler de manière plus souple avec les notions de citoyenneté linguistique et de voix.

De plus, afin d'appréhender la question identitaire des Franco-ontariens.nes, il s'avérera particulièrement utile de faire un tour d'horizon du contexte sociopolitique de l'époque. D'abord, le fait que ce documentaire sorte quatre ans après la fin de la Révolution tranquille (1959-1983 $\left.{ }^{\circ}\right)$ et sept ans après le premier référendum (mai 1980) en faveur de la souveraineté du Québec est particulièrement révélateur du malaise identitaire que ressentent les Ontariens et les Ontariennes de langue et/ou de culture canadienne-française. En nous plongeant au cœur de la problématique de l'éclatement du Canada français et de la dépossession de l'identité canadienne-française de ceux qui s'identifieront tour à tour comme Ontarois.es., Francoontariens.nes puis francophones (Gervais, 1995, p. 132) l'on se penche aussi sur l'évolution de la catégorisation des francophones en Ontario. On apprend que le seul critère retenu alors et ce jusqu'en 1986 dans la définition de francophone est exclusivement celui de la langue maternelle et correspond à la définition suivante : il s'agit de la « première langue parlée par le répondant et qu'il comprend encore le jour du recensement (je souligne). Comme le fait justement remarquer Mougeon (1980, pp. 41-42), ce critère pose un problème majeur dans la mesure où il englobe à la fois un continuum de personnes se déclarant « françaises » : certaines comprenant leur première langue de façon diverse tout en étant incapables de la parler alors que d'autres la comprennent parfaitement et la parlent constamment. Du point de vue linguistique, des auteurs comme Baudot et al. (1980, p. 8) remettent en question l'idéologie monolingue et ce dès les années 70-80:

(...) toute société comme tout individu évoluant et se transformant sans cesse suivant un double processus de différenciation et d'uniformisation, il est évident que ni une langue ni une personnalité individuelle ou collective ne sont réalités homogènes, monolithiques ou stables. Les équations qu'il est possible d'établir entre elles à certains moments de leur devenir ne tiennent pas à la nature des 
choses, mais sont la résultante de pratiques historiquement et socialement repérables. Aussi adoptet-on aujourd'hui une attitude plus prudente vis-à-vis de ces questions. On hésitera par exemple à prétendre que l'identité d'une personne est mutilée si elle passe par plus d'une langue. On ne proclamera plus si allègrement que la communauté de langue implique nécessairement le partage d'une même culture. On avancera même que, dans certaines conditions, le multilinguisme, loin de faire obstacle à l'unité nationale, peut contribuer puissamment à son maintien. Et on a cessé de croire à l'existence d'une langue qui serait l'expression parfaite et unique d'une identité donnée : on préfère plus justement parler de codes linguistiques correspondant à plusieurs types de comportement individuels ou collectifs, et comme eux en perpétuelle mouvance.

En prenant en compte le bilinguisme répandu chez les Franco-ontariens, des chercheurs tels que Baudot et al. (1980) (et d'autres) ont permis de démontrer l'importance de dépasser l'idéologie monolingue, basée sur une relation exclusive entre langue et nation (Byrd Clark et al., 2013), au profit d'une idéologie biplurilingue. En effet, le bilinguisme canadien, sorte de "monolinguisme dédoublé ou parallèle », selon Monica Heller (2002), a été nié voire diabolisé et s'est révélé être un véritable symbole d'acculturation et d'assimilation linguistique des francophones en situation minoritaire. Nous verrons à travers l'analyse du documentaire autobiographique d'auteure de 29 minutes « Deux voix comme en écho » que la réalisatrice, Claudette Jaïko s'inscrit particulièrement dans cette construction monolingue puisqu'elle met l'accent sur l'importance, en tant que Franco-ontarienne, de préserver sa langue et sa culture française en recréant, grâce à un ingénieux dialogue avec son frère Alain, les conditions de leur propre minorisation. II est à noter que ce positionnement monolingue sera fortement contesté une dizaine d'années plus tard notamment par la nouvelle francophonie ontarienne aux origines et aux répertoires identitaires diversifiés et notamment, africains, maghrébins, haïtiens, etc., artistes, gai.e.s et autres.

Le choix de ce film particulier s'appuie sur la nature même de la démarche de la réalisatrice : donner une voix à son frère, qu'elle considère " sans voix " puisqu' assimilé à la langue et à la culture anglaises. Ayant perdu sa voix française, Alain est invité par sa sœur, Claudette, à revisiter ce qu'elle croit être "leur» francophonie minoritaire. Réticent, Alain expose son point de vue d'assimilé assumé à la culture anglophone majoritaire et dominante de l'Ontario. Alors que Jaïko pense au départ prêter une voix aux sans voix, représentés majoritairement par son frère - mais aussi dans une certaine mesure par différents groupes autochtones ainsi que leur père, c'est la voix française de Claudette Jaïko qui va finir par s'effacer au fil de ce récit, finissant par utiliser l'anglais à la fin comme pour montrer sa double appartenance (francophone et bilingue) presque niée au cours de ce film. Il s'agit d'un témoignage bouleversant mais aussi porteur d'espoir si on le lit/lie à/avec une nouvelle grille de lecture empreinte de citoyenneté linguistique (Stroud, 2001; Lim et al., 2018). Ainsi, dans le contexte de ce film identitaire, peut-on parler d'actes de citoyenneté linguistique en tant que tels et si oui dans quelles mesures? De plus, quelles positions occupent respectivement Claudette Jaïko, son frère Alain ainsi que les différents membres de la famille Jaïko et les autres personnes évoquées? En d'autres termes, quels sont les divers degrés d'agentivité des acteurs.trices sociaux.les dans ce documentaire questionnant le caractère multifactoriel de l'identité franco-ontarienne et ses différents répertoires?

\section{Donner une voix aux sans-voix dans « Deux voix, comme en écho »: Des micro- actes de citoyenneté linguistique performés par qui?}

Avant de commencer l'analyse des différents micro-actes de citoyenneté linguistique utilisés par Claudette Jaïko tout au long de son court-métrage, nous aimerions tout d'abord nous intéresser au choix du titre de cette œuvre et à ce qu'il évoque. "Deux voix comme en écho " nous amène à nous questionner sur la pertinence du rapport entre la voix et l'écho, comme représentation de la voix dominante et de la voix dominée. En effet, selon le $\mathrm{TLFI}^{10}$ dans sa première acception, le terme « écho » renvoie à un " phénomène de réflexion d'une onde sur une surface ou sur des inhomogénéités existant dans le milieu de propagation; l'onde ainsi réfléchie » ou encore de « retour par réflexion d'un phénomène vibratoire ». Dans son emploi figuré, le terme peut aussi référer à un « propos exprimé, rapporté (...), souvent avec une connotation péjorative ». On compare ainsi l'écho à une imitation, une répétition, notamment quand " [une] personne (...) rapporte, répète les paroles d'autrui, ou (...) imite la conduite d'autrui »; on utilise alors des expressions telles qu' « en écho ». Cette locution adverbiale choisie par Jaïko réfère ainsi à « tout effet de résonnance ou de correspondance, de ressemblance ou de réponse ». Dans ce court-métrage, la notion d'écho semble s'appliquer de prime abord à Alain ainsi qu'à d'autres minorisé.e.s (en particulier les Autochtones). En outre, il est important également, pour les besoins de notre analyse, de garder en tête le fait que dans son utilisation à la tournure négative l'expression « ne pas rester sans écho » démontre un " accueil, [une] réaction favorable, [une certaine] sympathie voire une [franche] adhésion ». 
Le questionnement qui hante Jaïko et qui jalonne ce documentaire est : comment passe-t-on de minorisé.e.s à assimilé.e.s? L'expression de cette forme de minorisation extrême - ou assimilation - est tristement familière pour la réalisatrice puisqu'il s'agit de la voie qu'a empruntée son frère, Alain. En revanche, ce qui n'est pas clair pour elle sont les raisons voire les motivations qui l'ont poussé vers une vie dans la langue de la majorité. Ainsi, le point d'entrée du documentaire s'ouvre d'emblée sur les bords de la Baie Georgienne, l'origine, et consiste pour Jaïko à nous présenter sa vision d'une francophonie ontarienne minoritaire (pensée ici comme communauté homogène) sous la forme d'un récit qui s'amorce naturellement en français. La réalisatrice apparaît en gros plan, présage d'une introspection qui va suivre. Tel un écho (représentant métaphoriquement la voix étouffée et donc par extension le sort des minorisé.e.s) reprenant le bruit des vagues, d'une voix lente, douce et mesurée, elle commence son récit autobiographique :

Mes rêves et mes souvenirs sont en français.

Cette langue m'habite, me parle

S'il m'arrivait un jour de ne plus la parler

S'il m'arrivait un jour de ne plus l'entendre,

J'ai l'impression que moi-même, je ne serais plus qu'un rêve, qu'un souvenir. (Jaïko, 1987, Je souligne)

Le début de ce récit tant par le fond (le fait d'ouvrir ce film par la question linguistique et de parler français, se trouve dans l'identité francophone telle que perçue par Claudette Jaïko) que par la forme autodiégétique (accent mis sur le récit autobiographique et l'utilisation de la première personne "je ", répétitions et structures en miroir; voir Genette (1972) cité dans Lejeune, 1975/1996, p. 15) positionne d'emblée la réalisatrice. Elle fait tout de suite preuve d'une forme d'agentivité puisqu'elle fait automatiquement référence aux trois temporalités évoquées précédemment: le passé («Mes rêves et mes souvenirs sont en français »); le présent ( Cette langue m'habite, me parle ») et l'hypothétique assimilation exprimée par le conditionnel ("je ne serais plus qu'un rêve, qu'un souvenir »). En admettant qu'elle puisse perdre son français et devenir assimilée linguistiquement à la majorité anglophone, elle disparaîtrait du moins métaphoriquement. Les séquences qui vont suivre nous proposent des va-et-vient incessants entre l'enfance et la vie actuelle de la réalisatrice, comme autant de voyages entre ville (Toronto) et campagne (chez ses grands-parents), de passages entre modernité et tradition. De Toronto et de son enfance, elle nous dit :

On dit que le mot Toronto signifie lieu où la rivière rencontre le lac; là où tout se croise, se mêle et se perd. C'est ici que je suis née, que j'ai grandi et que je continue de vivre entourée de voix anglaises. S'il m'arrive parfois de m'y perdre alors une autre voix peu à peu me soulève et me dépose sur les plages sablonneuses de la Baie Georgienne. (Jaïko, 1987, Je souligne)

Ici, la réalisatrice se confie sur son expérience linguistique de francophone ( "une autre voix ») en milieu minoritaire urbain. En faisant référence à sa propre expérience de minorisée, elle avoue à mi-voix qu'elle s'est parfois « égarée » dans la multiculturelle Toronto (" où tout se croise, se mêle et se perd ») et que la seule façon de rester celle qu'elle a choisi d'être - une francophone - est pour elle de se remémorer l'origine: "les plages sablonneuses de la Baie Georgienne ». Cependant, à partir de 2'15, le bruit des vagues se fait entendre toujours plus fort comme pour suggérer un changement de perspective imminent. Le « T'en souviens-tu, Alain? » (2'43) claque alors tel un rouleau d'écume et ne va pas sans nous rappeler la devise québécoise "Je me souviens " à laquelle on s'attendrait en guise de réponse. Mais alors qu'un gros plan suggérant une nouvelle introspection nous présente Alain, la réponse tombe d'elle-même, en anglais, comme un faible écho, un vague souvenir (" only a memory ») rien de plus qu'une illusion: "Sometimes when I see something, I know that there is another word for it like seeing the world twice. But I know that it's only a memory, an illusion »(Je souligne). Les notions d'illusion et de souvenir, soulevées par la réponse d'Alain, nous rappelle la position d'Halbwachs (1950/1997) en ce qui a trait à la mémoire collective et à la faculté de se remémorer des souvenirs. À ce propos, cet auteur nous révèle que :

(...) nos sentiments et nos pensées les plus personnels prennent leur source dans des milieux et des circonstances sociales définis. (p. 65) [Ainsi,] pour évoquer de tels souvenirs, il faut que nous nous replacions au même instant dans des groupes qui n'ont l'un avec l'autre que des rapports rares et accidentels ( $p$. 81) (...) [car] ce qui leur manque précisément pour se comprendre, s'entendre et confirmer mutuellement les souvenirs de ce passé de vie commune, c'est la faculté d'oublier les barrières qui les séparent à présent. Un malentendu pèse sur eux, comme sur deux hommes qui se retrouvent et qui (...) ne parlent plus la même langue [littéralement ou pas] (p. 64).

Tout en initiant une sorte de dialogue avec son frère («T'en souviens-tu, Alain? »), Jaïko se positionne d'abord comme la voix dominante bien qu'elle tente d'inclure la voix (perçue comme) dominée de son frère dans son récit. De plus, la relative agentivité de Claudette - n'oublions pas qu'elle parle en français, langue certes officielle mais toutefois minoritaire en Ontario - s'exprime particulièrement lorsqu'elle présente sa 
vision - une vision qu'elle voudrait commune et homogène entre elle et son frère-de la réalité sociolinguistique complexe de la francophonie minoritaire de l'Ontario. Le but est donc pour Jaïko de déconstruire sa propre histoire afin d'essayer d'y impliquer et d'y inclure son frère et de mieux saisir les raisons voire les motivations qui l'ont poussé vers une vie dans la langue de la majorité. En effet, selon Parsons (1968), (cité dans Emirbayer et Mische, 1998) l'action humaine est mue par une volonté, une motivation telle que l'agentivité de l'acteur social s'oriente en fonction des valeurs qu'il s'est lui-même choisies. Dans sa démarche de mieux se comprendre elle-même, la réalisatrice entraîne Alain ainsi que, de manière secondaire, d'autres minorisé.e.s. Pour parvenir à porter la voix de ces sans-voix, la stratégie de Jaïko, au sens de Charaudeau (2004, p. 20) - « possible marge de manœuvre du sujet parlant » - consiste à utiliser le récit autobiographique et la mémoire collective, approche qui va lui permettre l'expression d'une certaine forme d'agentivité. Revenant sur l'éclairage théorique apporté par Halbwachs (1950/1997), nous sommes plus à même de comprendre la position d'Alain. En effet, ne s'identifiant plus comme faisant partie du groupe franco-ontarien, il n'est plus en mesure de se rappeler les souvenirs d'enfance et la culture familiale tels que les narre Claudette. Selon El Tibi (2001; cité dans Macé, 2017, p. 77),

(...) la notion originelle de culture renferme, (...) tout ce qui concerne l'évolution historique d'un peuple; c'est-à-dire, le passé commun d'une communauté réunie en une société distincte, véritable capital accumulé au fil des siècles (...) [et] qui contribue le mieux à l'identifier. Ce capital constitue en cela une mémoire commune. Dès lors qu'un membre s'en éloigne, il rejette l'appartenance à ce passé commun (...).

C'est ce qui semble être l'expérience vécue par Alain qui favorise son parcours sociolinguistique et culturel dans le monde de la majorité anglophone. Dumont (1995, pp. 17-18) ajoute que, la culture correspond, selon sa signification la plus large, à « (...) un stock de codes, de manière d'être et de faire indispensables à nos actions comme à l'existence en commun. (...) Un des aspects de la culture repose sur la question de l'héritage. Voilà en quoi elle pose, comme enjeu primordial, le problème de la mémoire » et donc l'incapacité pour Alain de se rappeler car il ne s'identifie plus à l'identité collective franco-ontarienne. L'assimilation semble ici avoir agi comme une sorte de trauma dans la mesure où elle empêche Alain, de manière consciente ou pas, d'accéder à cette part de souvenirs. On a ici affaire à la dimension subjective qui émerge lors de processus de reconstruction mémorielle ${ }^{11}$. En effet, la vision collective de la culture francoontarienne, embrassée par Claudette, s'apparente à une expérience et à un héritage communs alors que la vision individuelle, dont se réclame Alain, met davantage l'accent sur un devenir évolutif en fonction des différentes expériences socio-langagières et des stratégies et répertoires identitaires (Camilleri et al., 1990; Verhoeven, 2006; Macé, 2019, 2017) en résultant. Ainsi, une fois qu'on en hérite, il appartient à l'individu de choisir de continuer d'aller y puiser des éléments ou non. Ce qui caractérise Claudette Jaïko dans sa démarche c'est peut-être plus que tout son obstination à essayer de comprendre pourquoi son frère ne s'identifie plus comme francophone et surtout quelles sont les raisons de son assimilation. Alors que ce documentaire laisse, à première vue, apercevoir une relation de pouvoir asymétrique entre Jaïko et son frère, ce micro-acte de citoyenneté linguistique (la création d'un récit autobiographique en partie dialogique), va tout de même finir par permettre, en accédant et partageant certains de ses souvenirs enfouis, de doter progressivement Alain d'une certaine voix aux yeux de Claudette. La séquence sur les souvenirs de vacances (4'32) en est un parfait exemple. Alors que la caméra entraîne le/la spectateur.trice dans un champ de maïs où il/elle retrouve Claudette et Alain enfants vêtus de cirés jaunes, le récit reprend: " Je me souviens très bien de ces après-midis de vacances où nous jouions dans les champs de blés d'Inde. Mais à quoi jouions-nous au juste? Tout simplement à être heureux [éclats de rires des deux enfants] ». Le choix de la réalisatrice ici de retrouver un semblant de connivence à travers une même réaction (le rire des deux enfants) permet à Alain de se replonger dans un passé commun évoqué par la réalisatrice-narratrice.

Cependant, il va s'en suivre deux courtes séquences un peu ambivalentes mais qui semblent représenter l'état d'esprit menant au parcours identitaire d'Alain: la première séquence, nous présente Alain seul, toujours vêtu de son ciré jaune. II s'enfonce, tête baissée, dans le champ de maïs jusqu'à en sortir. Un plan très bref du village et de l'église suggère le manque d'attrait d'Alain pour ces lieux représentant la francophonie traditionnelle ontarienne. Le passage extrêmement rapide à la séquence suivante, où l'on retrouve les deux enfants à vélo dans la forêt, démontre la préférence d'Alain pour les balades à vélo dans la campagne avec sa sœur. L'atmosphère ressentie y est joyeuse. Un dialogue avec Alain commence véritablement à s'instaurer $\left(5^{\prime} 21\right)$ lorsqu'il commence à s'ouvrir et qu'il partage quelques-uns de ses souvenirs d'enfance : " I remember hot days in cool shade of the woods; Smell pine along the beach roads and soft white sand on the shallow water; Just being there! That's how we learn how fields and woods and beaches would be. Always. " L'utilisation de ce " always » marque l'ancrage permanent dans cette réalité concrète, paysagère et semble en totale opposition en regard aux réminiscences linguistiques limitées d'Alain. Peu à peu, cependant, c'est lorsque les souvenirs collectifs de veillées autour du feu avec leur oncle, qui leur racontait des histoires, émergent $\left(6{ }^{\prime} 03\right)$ que la réalisatrice effectue un changement de stratégie narrative : au lieu de poser une question à Alain, elle choisit d'amorcer le dialogue d'une manière quelque peu différente: "Toutes ces soirées, toutes ces histoires que notre oncle nous racontait » (je souligne), 
permettant ainsi à son frère de faire partie du récit et d'accéder ainsi à une certaine forme d'agentivité. À ce propos, il convient de revenir sur la perspective exprimée par Deumert (2018) selon laquelle la notion de citoyenneté linguistique devrait inclure « une perspective langagière (Languaging perspective), promouvant la langue comme mode d'action avec un sens situé et co-construit ${ }^{12} »$. En apparence, Jaïko semble être celle qui octroie une voix à son frère - ne fut-elle qu'en anglais - permettant ainsi à Alain de se remémorer, à sa façon, ses expériences et de relater sa propre perception de leurs souvenirs communs : "I never knew if he made them up; But some of those stories have taken place on those beaches; In those very woods; Waubashene; Cawaja; Ossasané; Nottawasaga, Penetanguishene ».

La narration des souvenirs d'Alain passe ainsi par l'énumération des lieux, "Waubashene; Cawaja; Ossasané; Nottawasaga, Penetanguishene ». Ces évocations amènent la réalisatrice à conclure $\left(6^{\prime} 45\right)$ en disant : "Parmi toutes ces histoires, celle que je préférais c'était celle des Hurons », comme pour ouvrir le dialogue à un récit historique parallèle, véritable écho; celui de l'acculturation et de l'assimilation des peuples autochtones au Canada. Le caractère dialogique de cet échange nous permet de nous questionner à nouveau sur la nature évolutive des souvenirs collectifs. Selon Halbwachs (1950/1997, p. 52) le fait de « penser et (...) [de] nous souvenir en commun nous permet d'enrichir notre expérience commune : « les faits passés (...) prennent (...) [ainsi] plus de relief " car nous avons évoqué ensemble diverses circonstances dont chacun de nous se souvient et qui « ne sont pas les mêmes bien qu'elles se rapportent aux mêmes événements ». Ainsi, sommes-nous en mesure de "revivre [ces événements] avec plus de force, parce que nous ne sommes plus seul[.e] à nous les représenter, et que nous les voyons maintenant, comme nous les avons vus autrefois, quand nous les regardions, en même temps qu'avec nos yeux, avec ceux d'un[.e] autre ». Les séquences qui suivent, principalement narrées par la réalisatrice, vont finir d'établir le parallélisme entre la disparition ou l'assimilation de certains peuples autochtones et l'assimilation des francophones en milieu minoritaire. Pour aller au bout de sa démonstration, la réalisatrice choisit de ne pas dialoguer avec son frère pendant environ 2'30 (soit de 6'45 à 9'17). L'absence de voix d'Alain est mise en avant à plusieurs reprises dans ce qui va suivre pendant environ une minute trente (9'23-11'07) et notamment lorsque Jaïko lance ce mini-dialogue:

- Alain, je n'entends plus ta voix ? Dis-moi quelque chose en français.

- I can't

- Tu ne veux pas?

- No it's not that. I don't even remember speaking it. And yet maman still speaks French to me and I understand. Maman never gives up. (Jaïko, 1987)

Ce dialogue est très représentatif de la divergence de parcours sociolinguistiques et culturels qui existe entre Alain et la réalisatrice. D'abord, Alain répond exclusivement en anglais hormis la référence " maman » ce qui montre le respect qu'Alain éprouve quant à la résilience de cette dernière : ("Maman never gives up »). II nous signale aussi en passant qu'il demeure maître de son destin puisqu'il est toujours en mesure de comprendre la langue française ( I understand ») et en ce sens, selon la définition de " francophone » du gouvernement de l'Ontario, il est et demeure francophone. L'utilisation de ce « never » semble reprendre en écho le terme («Always ») précédemment évoqué renforçant l'idée de permanence des paysages de son enfance qui viennent s'opposer à la disparition du français dans sa vie actuelle. Dans le cas d'Alain, il s'avère que seule la rupture avec la langue maternelle lui permet d'exprimer son identité : « (...) inconsciemment, (...) cette rupture avec la langue de [l]a mère représent[e] aussi pour (...) [lui] une autre façon de se définir, la possibilité d'adopter une identité autre que celle de sa propre mère » (Levaque, 2009, p. 52). Jaïko profite de l'incursion du mot maman en français pour revenir sur l'origine de leur famille (10'05) : « Maman est née à Lafontaine. Elle a toujours été fière d'être canadienne-française; elle a rencontré mon père un soir de bal quelque temps après qu'il eut immigré de France; lui il s'était fait à l'idée de vivre ici en anglais; la rencontre avec ma mère l'a pour ainsi dire obligé à renouer avec son pays. » Ce court récit familial nous indique que bien que la famille Jaïko soit endogame (leur mère est canadienne-française et leur père, né de parents ukrainiens, a grandi en France), le positionnement respectif de la mère et du père quant à la langue française est bien différent : alors que c'est quelque chose que la mère a à cœur, qui fait partie de son identité ( « fière d'être canadienne-française »), le père ne semble pas tenir tant que ça à son identité francophone (" lui il s'était fait à l'idée de vivre ici en anglais »). La mère en revanche tient à préserver le patrimoine linguistique et culturel familial notamment lorsqu'elle s'évertue à parler français avec son fils. Par sa nature, le récit biographique de la mère est suivi presque immédiatement par le récit biographique du père. Il est à noter que ce dernier est principalement narré par Alain. Les souvenirs qu'il a de son père nous font découvrir les similitudes et la connivence entre les deux hommes : l'acculturation et l'assimilation à la culture dominante. On va peu à peu découvrir à travers la voix d'Alain que le père a lui-même un héritage ukrainien qu'il a vite délaissé pour s'intégrer en France: " He once told me he never felt comfortable in expressing himself in his mother tongue ». Sur le contexte de l'assimilation, Kastersztein (1990, p. 35 cité dans Macé, 2017) nous rappelle que : 
Les acteurs sociaux impliqués vont non seulement tenter de faire admettre leur appartenance mais faire en sorte qu'elle ne puisse plus être remise en cause. Ils vont prétendre avoir oublié les caractéristiques historiques et culturelles qui les rendaient distincts et accepter l'ensemble des valeurs et des normes dominantes.

L'assimilation devient donc totalement assumée de la part d'Alain. Même s'il a choisi d'effacer la majorité des traits de sa culture d'origine, il semble sanctionné par sa sœur (voir Kastersztein, 1990). Ainsi, la voix dominante devient ici celle d'Alain même si la réalisatrice se permet quelques incursions sous un semblant de dialogue qui semble artificiellement maintenu. L'agentivité d'Alain permet non seulement à ce dernier de faire revivre son père en fonction de ses souvenirs mais aussi de diriger en quelque sorte la conversation : " Dad always knew what it looks like to be a stranger. His parents were Ukrainian when they settled in France when he was still very young. Like us, he had to deal with two languages: One at home and one in the outside ॥ (Je souligne). Les thématiques développées par Alain sont attendues; il s'agit des questions relatives aux difficultés liées au bilinguisme en milieu minoritaire, en particulier au fait de se sentir étranger dans sa langue maternelle et d'avoir à endurer un destin tout tracé. En effet, selon De Mattia-Viviès (2018, p. 8),

La langue (...) extérieure (...) permet d'échapper à la langue d'origine, vécue comme un entre-soi étouffant et (...) [un] anti-destin (...) permet[tant] d'entreprendre un travail de séparation d'avec la langue de l'intérieur d'origine, et d'échapper au traumatisme conflictuel entre langue de l'intérieur, de la faille, et langue de l'extérieur, de l'école.

Toutefois, l'utilisation du "like us », montre bien que c'est Alain qui détient les rênes à ce moment-ci, étant celui qui inclut sa sœur comme bilingue. Ce faisant, il fait montre d'un certain degré d'agentivité. Ce discours fait aussi ressurgir un souvenir particulier de la réalisatrice qui essaie de rectifier la trajectoire discursive de son frère, en le remettant sur la voie/voix : « Papa me disait souvent qu'il est important de savoir qui on est, d'où on vient ». Ce souvenir ne va pas être entendu ou intégré par Alain qui renchérit presque instantanément sur l'incapacité de son père à s'exprimer dans sa langue maternelle tout comme c'est le cas pour lui : " I have lots in common with dad. He once told me he never felt comfortable in expressing himself in his mother tongue, just as I never felt at ease in mine. When I heard it spoken, it sounds strange: foreigner and familiar at the same time ». Jaïko tente de rappeler à Alain que le français est langue familiale et langue officielle au Canada : "Mais le français n'est pas étranger ici ». La réponse cinglante ne tarde pas à se faire entendre : « It was for my friends. I don't hear it around me; it's a hidden culture ».

On apprend la similitude des parcours linguistiques entre Alain et son père et la difficulté pour ces derniers de se sentir à l'aise dans leur langue maternelle respective. Jaïko n'entend pas ce que lui dit son frère; elle ne semble pas admettre la réalité : "Tu te caches Alain, je le sais; tu le sais » (11'08). La structure en écho ( " je le sais; tu le sais ») essaie de rétablir la timide connivence instaurée précédemment mais n'y parvient pas. L'absence de réponse, telle une parole sans écho, incite la réalisatrice à continuer la conversation seule : « J'avoue que moi aussi j'ai parfois l'impression d'être étrangère à moi-même » (11'34). Ces divers échanges sont pertinents en cela qu'ils révèlent le clivage linguistique et la différence de perception identitaire au sein de cette famille. Même si on sent une certaine volonté de la réalisatrice - mais aussi du frère, en quelque sorte - de jouer le jeu en tentant d'établir un dialogue et de laisser les voix de chacun s'exprimer, cela ne se passe pas toujours de la manière espérée. Le malaise se fait encore plus profond lorsque Claudette tente de comparer son parcours linguistique à celui de son frère (qu'elle aurait souhaité similaire, soit dit en passant), notamment lors des séquences traitant de leurs expériences d'apprentissage de la langue française. Alors que les souvenirs de Claudette font de l'apprentissage du français quelque chose de naturel, de facile et d'agréable, l'expérience d'Alain est tout autre, comme en témoigne la séquence de l'école (14'03-14'46): "I used to get teased because of my French accent so I lost it and spoke like everybody else. By the time I was 8 or 9 I was thinking only in English. French becomes a struggle. I felt so inadequate! That's when I decided if I couldn't speak it well enough, I wouldn't even try ». L'explication quant aux raisons qui ont incité Alain à abandonner la langue française révèle un profond désarroi qui laisse Jaïko presque sans voix. Sa seule répartie : « Mais tu n'avais pas d'accent » est vite balayée du revers de la main par Alain lorsqu'il renchérit : "But I heard it. It's still there. Sometimes when I am tired, I have to watch it; have to be on my guard ». Ici, Alain souligne un point particulièrement important en ce qui concerne l'accent. En effet, l'accent est généralement perçu dans sa « fonction identitaire » (Léon, 1993, pp. 21-22) car il permet à un.e locuteur.trice de déterminer et d'accéder, par inférence, à l'identité sociolinguistique. Ainsi, la perception qu'a Alain de son propre accent l'empêche de se sentir francophone; comme on l'a vu ailleurs dans le récit; il se sent "inadéquat ", véritable imposteur dans sa langue maternelle. La séquence des devoirs (14'48-15'25) est un autre révélateur du décalage entre les expériences vécues par le frère et la sœur. Alors que Claudette attendait avec impatience la fin du souper pour la leçon quotidienne de français ("Le soir après le souper, maman et papa ressortaient leurs anciens livres d'école pour nous enseigner le français; j'aimais bien ces leçons »), le moment était redouté par Alain : " I used to hate those lessons ». À la nostalgie d'une époque révolue caractérisée par une vie rurale, catholique et en français vient s'opposer 
la promesse d'une vie dynamique, urbaine et en anglais. Une question que pose Alain à sa sœur et qui laisse démontrer l'agentivité dont il fait à présent preuve est la suivante : " - Why do you hang on to the past? - Parce qu'on se débarrasse jamais de son passé quoi qu'on fasse », lui répond-elle. Jaïko tente continuellement d'inclure son frère dans sa perception du passé : « Mon passé à moi, notre passé à toi et à moi, je ne peux pas y revenir par une seule route car ce passé est double, tout comme les îles et les lieux jadis ont été nommés deux fois comme en écho. Two names for everything (...). » (je souligne). Alain énumère alors les lieux dans les deux langues. Alors qu'Alain ne semble pas ému par la perte des vestiges du passé : "That's history. An ancient battle...someone else's fight. It doesn't touch me », la réaction de sa sœur est tout autre : « mais moi ça me touche! » (20’21)

Comme nous avons tenté de le démontrer au fil des exemples choisis, Alain, personnage présent à l'écran, accède progressivement à la parole par l'entremise de micro-actes de citoyenneté linguistique performés par la réalisatrice qui lui tend « le porte-voix » en lui posant des questions puis en l'invitant à faire partie du récit lui-même. La voix d'Alain peut désormais se faire entendre mais sera-t-elle vraiment écoutée notamment par sa sœur qui semble demeurer « sur sa faim » et quelque peu perplexe à la fin du courtmétrage? (21'14-22'01) :

N'est-il pas aussi issu de la même réalité que moi?

N'est-il pas lui aussi habité par la même inquiétude?

N'avons-nous pas eu la même enfance?

N'est-il pas encore mon frère?

Je ne lui reproche pas d'être devenu ce qu'il est mais de me laisser pour ainsi dire seule à la recherche de cette partie de moi-même qu'il nie et dont il partage pourtant le secret. (Jaïko, 1987, Je souligne)

La nostalgie est un thème récurrent pendant tout le documentaire, notamment à travers l'image lancinante des balançoires; tantôt occupées par les deux enfants, pour signifier le temps heureux et insouciant de l'enfance, les dernières séquences ne montrant plus qu'une balançoire occupée par Claudette puis une balançoire vide. Le temps de l'enfance semble aboli pour un temps. Pas tout à fait, le documentaire se termine à mi-voix avec la réalisatrice qui fredonne un air familier. Dans ce contexte, Claudette s'adresse pour la première et dernière fois en anglais à son frère (26'57 soit moins d'une minute avant la fin du documentaire; 27'20) comme pour s'avouer vaincue en signifiant son bilinguisme, sa double appartenance linguistique et culturelle. Elle insiste tout de même auprès de son frère pour qu'il chante avec elle :

- Don't you remember that song?

- No, it doesn't sound familiar.

- Maybe if you sing it with me, it would come back.

- No, I am a terrible singer.

- Come on. Let's try it.

[Claudette entame seule le début des paroles] - À la Claire fontaine...

C'est alors qu'Alain interrompt la chanson et que le court-métrage se conclut sur un martellement final comme pour signifier la dominance de sa voix de majoritaire anglophone : "No! I don't remember that at all » et comme pour marquer l'échec de cette dernière stratégie (chanter une chanson d'enfance propre au folklore francophone en particulier de l'Ontario) de la réalisatrice. Tout au long de ce documentaire, l'on peut s'interroger sur le rôle tenu par Claudette qui semble mettre l'accent et accorder beaucoup d'importance à l'incapacité qu'a son frère de s'identifier comme francophone et en particulier de parler français. Elle semble aussi mettre sous couvert ce qu'il a choisi de ne pas faire, notamment de parler français. On peut donc penser d'abord qu'Alain est simplement assimilé, mais l'on pourrait aussi se demander si Claudette Jaïko n'essaie pas de le forcer à se réassimiler à sa culture francophone effacée selon ses propres termes. Finalement, l'on pourrait se demander qui est finalement assimilé et à quoi? Et à partir de quel moment, peut-on véritablement parler d'assimilation? Ce court-métrage de Claudette Jaïko permet une prise de conscience du processus d'assimilation, qu'il soit assumé ou pas. Dans le cas d'Alain, son agentivité linguistique et sociale ne lui permet pas de s'exprimer en français et il parle donc en anglais retournant d'une certaine façon la situation; n'oublions pas qu'il s'agit de la langue dominante en Ontario. II peut agir de cette manière car son assimilation est un choix totalement assumé : "Already I could see that French wasn't going to be important to me. I didn't have to decide English was going to be the language of my world, the business world I wanted to live in $»$.

\section{Conclusion}

Cet article nous a permis d'explorer des exemples de micro-actes de citoyenneté linguistique en relation avec les concepts d'agentivité et de voix dans le contexte du cinéma identitaire et de la francophonie minoritaire en Ontario. Du point de vue de la réalisatrice, il semble qu'Alain se trouve dans une catégorisation 
rigide, un « état culturel permanent » de non francophone. En ayant exploré ce documentaire sous l'angle paradigmatique de la voix, les négociations identitaires d'Alain ont permis de mettre en avant un caractère identitaire plus dynamique. Tout au long de cette analyse, nous nous sommes efforcés de montrer que " détenir " ou " retrouver » cette voix pouvait avoir lieu à divers degrés en fonction des actions et des positionnements agentiques des acteurs.trices sociaux.ales. Le cheminement identitaire qui jalonne le documentaire de la réalisatrice Claudette Jaïko apparaît cependant comme non résolu à la fin du courtmétrage. II semblerait que cela fasse écho à un questionnement mettant en jeu la conviction de la réalisatrice en relation aux concepts de temporalité, de mémoire et de réalité, présent dès les premières minutes du film : « Dieu que j'aimerais, comme mon frère, pouvoir départager avec certitude le réel et l'illusoire, le passé, le présent, ce qui fuit et ce qui vient » (3'28-3'51).

S'avérant finalement plus complexe que le parcours identitaire de son frère-qui s'assume en tant qu'anglophone - le parcours de la réalisatrice semble encore semé d'embuches comme le montre la citation suivante : «Est-ce que je serai toujours ainsi un être double, mal défini, incapable de choisir entre la parole et l'écho? » (22'57-23'07) alors que deux représentations de l'identité linguistique franco-ontarienne s'opposent à la fin : d'un côté, la nostalgie d'une enfance perdue pour la réalisatrice Claudette Jaïko et de l'autre, un passage à l'âge adulte avec des contraintes identitaires assumées, pour Alain. Les deux dernières séquences se révèlent particulièrement signifiantes : " - Alain! Dis-moi que tout ceci existe, que je n'ai rien rêvé, que tu n'as rien oublié. (Je souligne); - You're not gonna find what you want from me. I am who I am. We're different. We're no longer children; you have to understand that. »

La question des parcours identitaires et des répertoires linguistiques n'est pas toujours une parole facile à libérer mais nous avons vu que cette dernière pouvait aussi s'exprimer d'une manière inattendue grâce à un nouveau regard sur les concepts d'agentivité, de voix et des micro-actes de citoyenneté linguistique tels que le récit autobiographique et le partage progressif de souvenirs collectifs. En résumé, l'agentivité implique différentes manières de vivre le monde, (...) elle s'applique à des situations dans lesquelles l'acteur.trice social.e entre en relation avec des personnes, des lieux, des significations et des événements environnants. (...) [Elle] implique également des interactions réelles avec ses contextes, dans quelque chose qui s'apparenterait à une conversation continue (...) « rempli de connotations dialogiques » (je traduis), comme une sorte de " maillon dans la chaîne de communication vocale » (Bakhtin 1986, p.91-92) ${ }^{13}$. Dans le processus d'agentivité, Emirbayer et Mische (1998, p. 973-974) soulignent l'importance de l'intersubjectivité, de l'interaction sociale et de la communication en tant que composantes critiques des processus agentiques: l'agentivité est toujours un processus dialogique par et à travers lequel les acteurs.trice immergé.e.s dans le passage temporel s'engagent avec les autres. Cette dernière phrase nous permet de rebondir sur un point : ce film met-il en scène un véritable "processus dialogique » tel que l'entendent Emirbayer et Mische (1988)? Patrick Charaudeau (2004, p. 19) nous permet de répondre du moins partiellement à ce questionnement :

(...) L'activité de relation à l'autre détermine un espace dans lequel le « Je » se trouve aux prises avec l'autre de la communication dans un rapport d'altérité intersubjective, un autre qui s'avère être [un « Tu » dans notre exemple]. Dans cet espace, il agit — ou est agi — [degré d'agentivité] en fonction de ce que sont les contraintes des dispositifs de communication dans lesquels il se trouve ([aussi appelées] les conditions situationnelles de la communication), et de la marge de manœuvre dont il dispose dans sa quête pour « s'individuer » (cette individuation serait-elle illusoire) (...)

Pour conclure, nous pensons que les exemples de micro-actes de citoyenneté linguistique dans le courtmétrage « Deux voix comme en écho » ne sont pas nécessairement ceux auxquels on s'attendait. Le recours à un cadre théorique mêlant les notions d'agentivité, de voix et de citoyenneté linguistique nous a permis de revisiter ce film identitaire sous une lumière différente car il permet plus de fluidité quant à l'expression de ce que l'on entend par « voix ». Comme mentionné chez Lim et al. (2018), le cadre théorique basé sur la notion de citoyenneté linguistique permet de redonner aux minorisé.e.s leurs voix en les considérant comme maître.esse.s de leur propre destin. En effet, contrairement à la notion de droit linguistique généralement utilisée dans le contexte législatif canadien, la notion de citoyenneté linguistique préconise le recours à de multiples formes langagières, y compris le fait de s'exprimer en anglais et en français sur des expériences s'étant déroulées dans des espaces temporels et sociolinguistiques francophones et/ou bilingues (anglais et langues autochtones) : « (...) pratiques qui [sont] mixtes, de bas statut ou transgressives - comme potentiellement pertinentes pour le bien-être social et économique, en acceptant qu'il est très difficile de prédire quoi que ce soit si l'on regarde simplement du centre ${ }^{14}$ ».

À l'effacement total de la voix de son frère, Claudette Jaïko nous montre, un peu malgré elle, la voie puisqu'en ayant eu recours à l'écho (voix minorisée), la voix de son frère qu'elle croyait pourtant éteinte ne reste pas sans écho même si elle s'exprime quasi-exclusivement en anglais (voix qui finit par être dominante). L'on peut interpréter ce changement de voix de la part de la réalisatrice (lorsqu'elle passe elle- 
même à l'anglais à la fin du documentaire) d'une part comme un échec mais aussi d'autre part comme une victoire partielle grâce à l'expression, chez Alain, d'une certaine forme d'agentivité.

\title{
Notes
}

\begin{abstract}
1 II est important de noter une évolution dans la définition de «francophone » au Canada entre les années 80 (date du film de Jaïko : 1987) et les années 2020 (année de l'article : 2022) notamment avec l'introduction par le gouvernement de l'Ontario de la définition inclusive de francophone (DIF) en 2009. Ainsi, alors que la définition classique utilisait le critère exclusif de langue "maternelle ", la définition inclusive de " francophone » identifie aussi comme francophones les allophones dont le français constitue la première langue officielle parlée ou utilisée couramment ou fréquemment. Ainsi, les chiffres de recensement à partir des années 2010 reflètent ce changement de définition.
\end{abstract}

2 « (...) as a blueprint for a conceptual space within which to think differently (...) about language and ourselves (Ibid. 18) [that] might comprise an empowering politics of language for agency and change » (Ibid., p. 3).

${ }^{3}$ « (...) through the lens of citizenship and participatory democracy »

${ }^{4}$ « (...) alternative forms of being-together-in-difference (...) in reconstruct[ing] [and in favouring] the multilingual encounters as a moment for the celebration of difference rather than the suppression of voice (...)».

${ }^{5}$ « (...) as a temporally embedded process of social engagement, informed by the past (in its « iterational » or habitual aspect) but also oriented toward the future (as a " projective " capacity to imagine alternative possibilities) and toward the present (as a " practical-evaluative " capacity to conceptualize past habits and future projects within the contingencies of the moment) " (Emirbayer et Mische, 1998, p. 962; je traduis).

${ }^{6}$ « (...) gain crucial analytical leverage for charting varying degrees of maneuverability, inventiveness, and reflective choice shown by social actors in relation to the constraining and enabling contexts of action " (Emirbayer et Mische, 1998, p. 964; je traduis).

${ }^{7}$ Voir Emirbayer et Mische, 1998, pour plus de détails.

8 « (...) the capacity of human beings to shape the circumstances in which they live » (Emirbayer et Mische 1998, p. 965; je traduis).

9 II est admis que la Révolution tranquille québécoise s'étend des années 60 aux années 80 . Les historiens la font généralement débuter le 7 septembre 1959 (mort de Maurice Duplessis) et terminer le 16 février 1983 (adoption de la loi dite matraque; le gouvernement du Parti québécois oblige alors le retour au travail des grévistes des écoles et collèges du secteur public) (Pâquet et Savard, 2021).

10 Trésor de la Langue Française Informatisé.

11 Pour plus de détails sur cette question, voir Halbwachs, 1992 mais aussi Mead, 1932, p. 12 (cités dans Emirbayer et Mische, 1998).

12 « (..) a languaging perspective promoting language as a mode of action with meaning being (...) situated and co-constructed » (Deumert dans Lim et al., 2018, p. 289; je traduis).

${ }^{13}$ Cité dans Emirbayer et Mische, 1998; je traduis.

14 « (...) practices that [a]re mixed, low-status or transgressive - as potentially relevant to social and economic well-being, accepting that it is very hard to predict any of this if one is merely watching from the centre » (Rampton et al., 2018, p. 70; je traduis). 
Macé, Fanny. « Agentivité et citoyenneté linguistique de la francophonie en Ontario. » Nouvelle Revue Synergies Canada, N 15 (2022).

\section{Bibliographie}

Aquin, H. (1962). La fatigue culturelle du Canada français. Liberté, 4(23), 299-325. URL : https://id.erudit.org/iderudit/59892ac.

Bakhtin, M. M. (1986). The problem of speech genres. Dans C. Emerson et M. Holquist (eds.), Speech genres and other late essays (p. 60-102). V. W. McGee (trad.). University of Texas Press.

Baudot, A., Jaubert, J.-C. et Sabourin, R. (1980). Actes du 3e colloque sur l'identité culturelle et la langue française dans les Amériques (ICFA III; Toronto, 2-5 juin 1976). Ottawa : Centre international de recherche sur le bilinguisme, Québec : Université Laval.

Bell, A. (2017). Giving voice: A personal essay on the Shape of Sociolinguistics. Journal of Sociolinguistics, 21 (5), 587-602. DOI:10.1111/josl.12268.

Byrd Clark, J., Lamoureux, S. et Stratilaki, S. (2013). Apprendre et enseigner le français dans l'Ontario, Canada : Entre dualité linguistique et réalités plurielles et complexes. Les Cahiers de l'Acedle, 10 (3). DOI : https://doi.org/10.4000/rdlc.2603.

Camilleri, C., Kastersztein, J., Lipiansky, E.-M., Malewska-Peyre, H., Taboada-Leonetti, I. et VasquezBronfman, A. (dir.). (1990). Stratégies identitaires. Presses Universitaires de France (PUF).

Charaudeau, P. (2004). Tiers, où es-tu? La voix cachée du tiers : Des non-dits du discours. L'Harmattan.

Corbeil, J. P. et Lafrenière, S. (2010). Portrait des minorités de langues officielles au Canada: Les francophones de l'Ontario. (89-642-X au catalogue). Statistique Canada. https://www150.statcan.gc.ca/n1/pub/89-642-x/2010001/article/section1-fra.htm

De Mattia-Viviès, M. (2018). Entrer dans la langue ou dans les langues : De la langue maternelle à la langue « mat-rangère ». E-rea [En ligne], 16 (1). DOI : https://doi.org/10.4000/erea.6502.

Deumert, A. (2018). Commentary - on participation and resistance. Dans L. Lim, C. Stroud et L. Wee. (eds.). The multilingual citizen: Towards a politics of language for agency and change (p. 289-299). Multilingual Matters'.

Dumont, F. (1995). L'avenir de la mémoire. Nuit Blanche.

Écho. (s. d.). Dans TLFI (Trésor de la Langue Française Informatisé). http://stella.atilf.fr/Dendien/scripts/tlfiv5/affart.exe?44;s=2189268075;?b=0

El Tibi, Z. (2001). La Francophonie et le dialogue des cultures. L'Âge d'homme.

Emirbayer, M. et Mische, A. (1998). What is Agency? American Journal of Sociolinguistics, 103 (4), 9621023.

Genette, G. (1972). Figures III. Éditions du Seuil.

Gervais, G. (1995). Aux origines de l'identité franco-ontarienne. Cahiers Charlevoix, 1, 125-168. https://doi.org/10.7202/1039462ar.

Gouvernement de l'Ontario Ministère des Affaires francophones. (2019). Profil de la population francophone de l'Ontario - 2016. https://www.ontario.ca/fr/page/profil-de-la-population-francophone-de-lontario-2016.

Halbwachs, M. (1997). La mémoire collective. Albin Michel. (Travail original publié en 1950).

Halbwachs, M. (1992). On Collective Memory. (L. A. Coser dir. et trad.). University of Chicago Press.

Heller, M. (2002). Éléments d’une sociolinguistique critique. Didier.

Jaïko, C. (1987). Deux voix comme en écho [Film]. Office national du film (ONF). 
Kastersztein, J. (1990). Les stratégie identitaires des acteurs sociaux: Approche dynamique des finalités. Dans C. Camilleri, J. Kastersztein, E.-M. Lipiansky, H. Malewska-Peyre, I. Taboada-Leonetti, et A. Vasquez (dir.), Stratégies identitaires (p. 27-41). Presses Universitaires de France.

Lejeune, P. (1996). Le pacte autobiographique. Éditions du Seuil. (Travail original publié en 1975).

Léon, P. (1993). Précis de phonostylistique. Nathan.

Levaque, C. (2009). La langue: Délaissée, rejetée, oubliée et retrouvée. Quelques réflexions, quelques questions. Filigrane, 18 (2), 51-69. https://doi.org/10.7202/039289ar.

Lim, L., Stroud, C. et Wee, L. (dir.). (2018). The multilingual citizen: Towards a politics of language for agency and change. Multilingual Matters.

Macé, F. (2019). Perspectives transculturelles et stratégies identitaires des locuteurs de français à Calgary. The Representation of the Other/La Représentation de I'Autre, Otherness: Essays and Studies, 7.2, 145-170. Otherness.dk.

Macé, F. (2017). Stratégies et constructions identitaires des locuteurs de langue française en Alberta : Évolution de la référence nominative. [Thèse de doctorat inédite, Werklund School of Education, University of Calgary].

Mead, G. H. (1932). The Philosophy of the present. University of Chicago Press.

Miville, S. (2013). Le cinéma identitaire franco-ontarien: Un cas de figure de la fatigue culturelle. Voix Plurielles, $10(1), 2-18$.

Mougeon, R. (1980). Le maintien du français en Ontario. Dans A. Baudot, J.-C. Jaubert et R. Sabourin, Actes du 3e colloque sur l'identité culturelle et la langue française dans les Amériques (ICFA III; Toronto, 2-5 juin 1976, 41-50). Ottawa: Centre international de recherche sur le bilinguisme et Québec: Université Laval.

Mougeon, R. et Béniak, É. (1994). Bilingualism, language shift, and institutional support for French: The case of the Franco-Ontarians. Int'l J Soc Lang,105/106, 99-126.

Pâquet, M. et Savard, S. (2021). Brève histoire de la Révolution tranquille. Éditions du Boréal.

Parsons, T. (1968). The Structure of Social Action, 2 vols. Free Press.

Rampton, B., Cooke, M. et Holmes, S. (2018). Sociolinguistic Citizenship. Journal of Social Science Education, 4. DOI: 10.4119/JSSE-1097.

Scott, J. (2009). The Art of not being governed: An anarchist history of upland southeast Asia. Yale University Press.

Sing, P. et Dansereau, E. (Dir.). (2012). Impenser la francophonie : Renouvellement, recherches, diversité, identité..., [Actes du $22^{\mathrm{e}}$ colloque du CEFCO], University of Alberta.

Statistique Canada. (Mai 2020). Tableau 17-10-0009-01. Estimations de la population, trimestrielles. DOI : https://doi.org/10.25318/1710000901-fra.

Stroud, C. (2001). African mother-tongue programmes and the politics of language: Linguistic citizenship versus linguistic human rights. Journal of Multilingual and Multicultural Development, 22(4), 339355.

Verhoeven, M. (2006). Stratégies identitaires de jeunes issus de l'immigration et contextes scolaires : vers un renouvellement des figures de la reproduction culturelle. Éducation et francophonie, 34(1), 95110.

Voix. (s. d.). Dans TLFI (Trésor de la Langue Française Informatisé). http://stella.atilf.fr/Dendien/scripts/tlfiv5/visusel.exe?15;s=2239403115;r=1;nat=;sol=4 